\title{
GESTORES EXCESSIVAMENTE CONFIANTES E OTIMISTAS GERENCIAM RESULTADOS? EVIDENCIAS EM COMPANHIAS LISTADAS NA B3
}

\author{
Allan Marcelo de Oliveira \\ Rodrigo Oliveira Soares ${ }^{2}$
}

\begin{abstract}
Resumo: Este artigo objetiva investigar a influência dos vieses cognitivos excesso de confiança e otimismo sobre o gerenciamento de resultados. Utilizou-se uma amostra composta por 136 companhias listadas na B3 no período entre 2011 e 2015. Os dados foram coletados no Bloomberg ${ }^{\circledR}$ e foi utilizada uma regressão pooled OLS com auxílio do software STATA ${ }^{\oplus}$. A variável dependente, Gerenciamento de Resultados, foi estimada pelo modelo de Jones Modificado (Dechow, Sloan \& Sweeney, 1995), e a independente, excesso de confiança e otimismo, com adaptação de uma medida proposta por Malmendier e Tate (2005). Os resultados sugerem que o excesso de confiança e o otimismo estão relacionados positiva e significativamente com o gerenciamento de resultados, ou seja, CEOs mais excessivamente confiantes e otimistas gerenciam mais seus resultados. Os resultados contribuem para um melhor entendimento das motivações para o gerenciamento de resultados, bem como para instigar novas pesquisas que aprofundem a influência de aspectos comportamentais sobre a tomada de decisão da gestão.
\end{abstract}

Palavras-chave: Excesso de Confiança e Otimismo. Gerenciamento de Resultados. Finanças Comportamentais.

am.oliveira9490@gmail.com - Universidade Federal do Paraná

${ }^{2}$ rosoares@ufpr.br - Universidade Federal do Paraná

- DOI: http://dx.doi.org/10.14392/asaa.2018110303

- Artigo submetido em: 03/04/2018. Aceito em: 07/02/2019. 


\title{
DO OVERCONFIDENT AND OPTIMISTIC CEO'S MANAGE EARNINGS? EVIDENCES IN COMPANIES LISTED AT B3
}

\begin{abstract}
This paper aims to investigate the influence of the cognitive biases overconfidence and optimism on earnings management. We worked with a sample of 136 firms listed at B3 between the years 2011 and 2015. The Data source was the Bloomberg ${ }^{\circledR}$ database and we performed a pooled OLS regression using STATA ${ }^{\oplus}$ software. The dependent variable (Earnings Management) was estimated using the Modified Jones Model (Dechow, Sloan \& Sweeney, 1995), and the independent one (overconfidence and optimism), was an adaptation of the measure proposed by Malmendier and Tate (2005). The results points that overconfidence and optimism are positive and significantly related to earnings management, that is, more overconfident and optimistic CEOs are more likely to manage earnings. Our findings contribute both, to better understanding of the motivations to earnings management and to instigate new research that deepens the influence of behavioral aspects on management decision making.
\end{abstract}

Keywords: Overconfidence and Optimism. Earnings Management. Behavioral Finance. 


\section{INTRODUÇÃO}

$\mathrm{T}$ rabalhos basilares da Teoria de Finanças, como Markowitz (1952, 1959), Modigliani e Miller (1958), Sharpe $(1963,1964)$ além de Fama (1975) compartilham, dentre seus pressupostos básicos, que os agentes utilizam uma função de utilidade para maximizar seu bem-estar, são avessos ao risco e racionais (Castro Junior \& Famá, 2002). Esses estudos apresentam diversas abordagens que buscam resolver problemas específicos, mas desconsideram a limitação da racionalidade dos agentes, baseando-se no paradigma da racionalidade.

Por sua vez, no contexto das decisões econômicas, a capacidade de o indivíduo tomar decisões racionais é discutida pelo menos desde Simon (1957), que na sua Teoria da Racionalidade Limitada discute limites à racionalidade, bem como a contribuição da psicologia para a melhor compreensão do comportamento do decisor (Famá, Cioffi \& Coelho, 2008).

Considerando então a racionalidade como uma premissa básica para a Teoria tradicional de Finanças, e por outro lado as contribuições, tanto de Simon quanto dos estudos da área de psicologia que apontam que o ser humano apresenta desvios quanto ao comportamento racional esperado, desenvolveu-se Finanças Comportamentais, incorporando aspectos psicológicos dos indivíduos de forma a aprimorar a Teoria Tradicional de Finanças, (Halfeld \& Torres, 2001; Kimura, 2003; Silva, Piccoli, Cruz \& Clemente, 2014).

Segundo Yoshinaga e Ramalho (2014) o trabalho seminal das Finanças Comportamentais foi o dos psicólogos israelenses Kahneman e Tversky (2013). Neste estudo ao criticar a racionalidade, os autores supõem que os investidores adotam heurísticas e processos mentais enviesados, baseando-se em estereótipos (conservadorismo, excesso de confiança, exibicionismo, entre outros) levando a incompatibilidades com a Hipótese de Mercados Eficientes - HME (Kimura, 2003). Diversos padrões de comportamento são analisados desde então, considerando atalhos mentais (heurísticas) e desvios do comportamento esperado (vieses), na área de Finanças Comportamentais, tais como: representatividade, efeito manada, aversão à perda, ancoragem, Além do excesso de confiança e otimismo, que são o foco deste trabalho.

Por sua vez, o gerenciamento de resultados surge do comportamento oportunístico do gestor, ou seja, quando há julgamento sobre as informações financeiras a fim de manipular resultados contábeis, iludindo assim investidores sobre o desempenho econômico das empresas (Healy \&Wahlen, 1999). Dado que o lucro reportado afeta o processo decisório de investidores e credores, pode-se argumentar que os gestores tendem a manipular resultados de acordo com o comportamento esperado destes usuários, em relação às informações divulgadas. Logo é importante detectar determinadas atitudes da gestão para redução uma assimetria informacional. Considera-se que a qualidade da informação contábil é essencial para a tomada de decisão nas organizações.

Segundo Cavalheiro (2015) diversos pesquisadores têm voltado sua atenção para a compreensão do indivíduo e seu processo de tomada de decisão financeira. $\mathrm{O}$ autor critica que o comportamento financeiro está pautado em pressupostos contrários aos da Teoria Tradicional de Finanças indicando que pesquisadores deveriam incluir em suas análises, variáveis comportamentais.

De acordo com Halfeld e Torres (2001), os administradores e investidores de mercado, devido às crenças que têm em suas habilidades aliadas à formação acadêmica, estão induzidos a um comportamento autoconfiante e otimista. Por sua vez, Kermani, Kargar e Zarei (2014) apontam que, sendo os gestores excessivamente confiantes e otimistas, e dado que estes têm acesso aos resultados da empresa, podendo 
medir sua eficiência antes da divulgação efetiva, pode-se esperar que, se eles não conseguem atingir o lucro e a eficiência desejada, tenderiam a manipular resultado.

Diante disto, propõe-se a seguinte questão: qual a relação entre o excesso de confiança e o otimismo dos gestores sobre o gerenciamento de resultados em companhias listadas na B3?

Com o desejo de responder à questão de pesquisa, o objetivo geral é verificar a influência do excesso de confiança e otimismo sobre o gerenciamento de resultados em companhias listadas na B3 no período de 2011 a 2015.

No processo de tomada de decisão empresarial, estudar o efeito do excesso de confiança e otimismo é relevante dado que, para tomar decisões complexas e que envolvam risco os indivíduos utilizam de atalhos mentais (Kahneman \& Tversky, 1974) e tendem a de fato ser excessivamente confiantes e otimistas (Kahneman \& Tversky, 1974; Hackbarth, 2008). Outra evidência que motiva esse estudo é que segundo Hackbarth (2008), gestores mais otimistas apresentam a tendência de acreditarem que suas decisões financeiras são melhores do que de fato são. Ainda neste sentido, Schrand e Zechman (2012) argumentam que as expectativas excessivamente otimistas sobre o desempenho futuro levam os gestores a se envolverem involuntária ou intencionalmente com gerenciamento de resultados.

Neste contexto, esta pesquisa contribui com aspectos teóricos relacionados ao tema, buscando descrever e identificar a influência dos vieses de excesso de confiança e otimismo sobre o gerenciamento de resultados em companhias listadas na B3. Pode-se argumentar que a pesquisa sobre gerenciamento de resultados e qualidade da informação contábil tem se embasado principalmente em aspectos relacionados a conflitos de interesse, assimetrias de informação e oportunismo, e os aspectos comportamentais têm potencial para explicar tais práticas. Por fim, sua contribuição social e prática deve-se ao fato de explorar empiricamente o contexto das ações comportamentais influenciando práticas gerenciais, propiciando aos investidores e stakeholders, informações relevantes para o processo de tomada de decisão. Assim, o mercado deve buscar considerar a influência de tais vieses nos gestores ao processar as informações relevantes para as tomadas de decisão.

Este artigo está estruturado em cinco seções: após a introdução são apresentados na sequência a fundamentação teórica, aspectos metodológicos, apresentação e discussão dos resultados, e finalmente a conclusão.

\section{FUNDAMENTAÇÃO TEÓRICA}

\subsection{Gerenciamento de Resultados}

Empresas podem optar por práticas diferentes mesmo quando estão tratando de um mesmo evento econômico, graças a vazios nas normas contábeis e legislação tributária (Cabello, 2015). Pode-se usar como exemplo, dentre as práticas contábeis voluntárias: métodos de depreciação (linear, com base na vida útil do bem, versus acelerada, com base no desgaste do bem); método de controle de estoque (Primeiro que Entra, Primeiro que Sai - PEPS versus Último que Entra, Primeiro que Sai - UEPS versus Custo Médio Ponderado Fixo - CMPF); apropriação de juros (ativo ou despesa); dentre outros.

Silva, Martins e Lemes (2016) apontam que as pesquisas que estudam fatores motivacionais para o gerenciamento de resultados nem sempre são conduzidas sob a perspectiva racional (Carpenter \& Feroz, 2001; Jaafar \& Mcleay, 2007; Collin, Tagesson, Andersson, Cato \& Hanson 2009; Cole, Branson \& Breesch, 2013; Haller \& Wehrfritz, 2013; Nobes \& Perramon, 2013; Stadler \& Nobes, 2014; Pinto, Martins \& Silva, 2015). Isso se dá, segundo os autores, porque a pressão institucional pode influenciar preferências e limitar a capacidade cognitiva, trazendo como consequências limites à capacidade de escolhas totalmente racionais na Contabilidade. 
A prática do GR acontece por meio da manipulação de accruals ou acumulações totais, que são definidos como sendo a diferença entre o resultado contábil e o fluxo de caixa da empresa. Martinez $(2008$, p. 8) cita que os accruals dizem respeito às contas de resultados que compõem o lucro, mas que não implicam em movimentação de disponibilidades. Isto se deve ao registro das informações sob o Regime de Competência, em que são feitos os registros das receitas e despesas, independentemente do seu efetivo recebimento e pagamento, em contraponto ao Regime de Caixa.

Assim, através dos accruals é possível manipular as informações financeiras com a finalidade de, por exemplo, apresentar aos investidores um lucro maior. Os accruals podem ser separados em discricionários e não discricionários. Os últimos são relacionados às condições do próprio negócio, sem julgamento; já os discricionários refletem as ações de escolhas da administração, relacionadas ao GR.

De maneira geral os modelos de mensuração de gerenciamento de resultados seguem as seguintes etapas: i) levantar os accruals totais da empresa no período $t$ em função das variáveis que representam os fatores relevantes que afetam o comportamento dos accruals totais; ii) apurar os accruals não discricionários com base nos coeficientes encontrados e nos valores reais das variáveis explicativas e; iii) estimar os accruals discricionários pela diferença entre os accruals totais e os accruals não discricionários (Consoni, 2014).

\subsection{Excesso de confiança e otimismo}

Estudos indicam que o excesso de confiança e otimismo são características comuns dos gestores e que esses vieses cognitivos afetam suas decisões econômicas reais (Roll, 1986; Malmendier \&Tate, 2005; Felleg, Moers \& Renders, 2012).

O excesso de confiança também é definido como um erro sistemático de julgamento, no qual as pessoas superestimam sua própria acuidade (Schaefer, Williams, Goodie \& Campbell, 2004; Dittrich, Guth \& Maciejovsky, 2005) e a precisão do conhecimento (Dittrich et al. 2005). Portanto este viés refere-se à tendência de o indivíduo superestimar seus conhecimentos, atributos e características, podendo levá-lo a pensar que é mais competente e habilidoso do que realmente é.

Heaton (2002) aponta que os gestores são otimistas quando, sistematicamente, superestimam a probabilidade de um bom desempenho da empresa e subestimam a probabilidade de um mau desempenho.

A pesquisa em finanças, entretanto, tem ignorado a influência de vieses comportamentais na modelagem do complexo processo decisório em ambientes organizacionais (Hackbarth, 2009). Uma das dificuldades reside em medir estes vieses e heurísticas, o que não é uma tarefa simples, pois tais comportamentos não são diretamente observáveis em informações públicas. Pesquisas têm tentado criar modelos com proxies que indiquem a presença destes vieses, entretanto não há na literatura consenso quanto à validade dos modelos.

Dos modelos apresentados na literatura, destacam-se as pesquisas de Malmendier e Tate (2005; 2008), que construíram medidas de excesso de confiança e otimismo com base nas decisões pessoais das carteiras dos diretores. Os autores assumem que gestores excessivamente confiantes adquirem maior número de ações da companhia, dado que estão confiantes na sua capacidade de aumentar o valor das ações, calculando por meio da variação líquida das transações dos diretores com ações da própria companhia (Hribar \& Yang, 2015). Esta pesquisa utiliza-se desta definição operacional, com alterações a serem apresentadas no próximo capítulo.

Embora o excesso de confiança e o otimismo sejam distintos, trabalhos da área de psicologia e outras assumem que eles estão estreitamente relacionados (Taylor \& Brown, 1988; Barros \& Silveira, 2008), 
sendo que diversas pesquisas não têm dissociado estes vieses (Barros \& Silveira, 2008, Malmendier \& Tate, 2008, Li \& Hung, 2013) tratando-os de forma conjunta devido a isto. Quando Malmendier e Tate (2005) elaboraram uma medida denominada Net Buyer eles a usaram para mensurar o excesso de confiança exclusivamente. Entretanto, os autores utilizaram a mesma medida posteriormente (Malmendier \& Tate, 2008), interpretando-a como uma mensuração para indivíduos "excessivamente confiantes" ou "otimistas". O corrente trabalho de pesquisa apoia-se nesta unidade pois o fato de o CEO tender a uma subdiversificação devida a uma concentração acionária na própria empresa ('compra líquida' positiva) indica não só uma confiança excessiva sobre as próprias habilidades, mas também otimismo quando subestimam a probabilidade de eventos adversos em variáveis exógenas. Desta forma, esta variável atua como proxy indistintamente, tanto para o excesso de confiança quanto para o otimismo, sendo impossível a separação dos efeitos, assim como nas pesquisas de Barros \& Silveira (2008), Malmendier \& Tate (2008), Li \& Hung (2013).

\subsection{Trabalhos Correlatos}

Segundo Cavalheiro (2015) diversos pesquisadores têm voltado sua atenção para a compreensão do indivíduo e seu processo de tomada de decisão financeira. $\mathrm{O}$ autor critica que o comportamento financeiro está pautado em pressupostos que excedem os limites da racionalidade esperada, contrários aos da Teoria Tradicional de Finanças, indicando que pesquisadores deveriam incluir em suas análises, variáveis comportamentais.

A literatura nacional existente sobre gerenciamento de resultados se manteve crescente desde o início do século, com diversos estudos empíricos que adicionam fatores e resultados a literatura da área como: Martinez (2001), Paulo (2007), Baptista (2008), Nardi e Nakao (2009), Avelar e Santos (2011), Dallabona, Martins e Klann (2014), Reis, Lamounier e Bressan (2015), Martins, Paulo e Monte (2016), Mazzioni, Prigol, Moura e Klann (2016), entre outros. Apesar de crescente não foi encontrado na literatura nacional estudos que incorporem variáveis baseadas em vieses cognitivos dos gestores.

Por outro lado, na literatura internacional é possível verificar estudos como o de Ge, Matsumoto, e Zhang (2011), Hilary e Hsu (2011), Schrand e Zechman (2012), Bouwman (2014), Kermani et al. (2014), entre outros, que em diferentes populações testaram a influência de vieses, mais especificamente nestes casos o excesso de confiança e otimismo, sobre as decisões financeiras e a prática de gerenciamento de resultados. Nota-se portanto, na escassez de estudos que trabalhem vieses cognitivos e gerenciamento de resultados em âmbito nacional, a oportunidade para a verificação da influência de vieses sobre o gerenciamento de resultados em companhias brasileiras.

\section{METODOLOGIA}

\subsection{Dados e Amostra}

Para identificar a influência dos vieses comportamentais, excesso de confiança e otimismo, sobre o gerenciamento de resultados de companhias listadas na B3, a pesquisa caracteriza-se quanto ao objetivo, como descritiva e explicativa (Gil, 2002), em relação à abordagem do problema, caracteriza-se como quantitativa (Martins, 2002) e no que se refere à técnica de coleta de dados a pesquisa classifica-se como documental (Marconi \& Lakatos, 1991).

A população a ser estudada foi composta por todas as companhias listadas na Brasil, Bolsa, Balcão (B3), utilizando o corte longitudinal de 2011 a 2015, correspondendo a 418 empresas.

Para definição da amostra da pesquisa, foram excluídos inicialmente os bancos, companhias de seguros e holdings, (143 empresas) devido a apresentarem diferentes peculiaridades quanto à mensu- 
ração de variáveis, como alavancagem financeira e rentabilidade por exemplo. Também foram excluídas 139 empresas que não apresentaram todos os dados para levantamento das variáveis fundamentais da pesquisa, especialmente a mensuração da variável de excesso de confiança e otimismo. Cabe ressaltar também que o intervalo de tempo ficou reduzido a 2011-2015, pois na construção da variável referente ao excesso de confiança e otimismo (ECO) os anos de 2010 e anteriores apresentaram dados referentes às transações dos CEO para menos de 30 das empresas listadas, fato que prejudicaria o tratamento estatístico. Deste modo, a amostra da pesquisa foi composta por 136 empresas.

Para se efetuar a coleta de dados e construção das variáveis, o primeiro passo foi o levantamento de dados financeiros, realizado por meio da base de dados Bloomberg ${ }^{\circledR}$, tais como variáveis contábeis e informações referente às transações com ações das companhias que apresentavam informação suficiente para as variáveis do estudo. A seguir são apresentadas descrições detalhadas de cada uma das variáveis utilizadas no estudo.

\subsection{Definição de Variáveis}

O Gerenciamento de resultados foi mensurado mediante a estimação (proxy) dos accruals discricionários, sendo apurada pela diferença entre: i) accruals totais, obtido por meio do enfoque do Balanço e, ii) accruals não discricionários, obtido por meio do modelo Jones Modificado (Dechow et al., 1995) apresentado a seguir:

$$
\mathrm{TA}_{\mathrm{it}}=\left(\Delta \mathrm{AC}_{\mathrm{i}, \mathrm{t}}-\Delta \operatorname{Disp}_{\mathrm{i}, \mathrm{t}}\right)-\left(\Delta \mathrm{PC} \mathrm{C}_{\mathrm{i}, \mathrm{t}} \Delta \operatorname{Div}_{\mathrm{i}, \mathrm{t}}\right)-\mathrm{DDA}_{\mathrm{i}, \mathrm{t}} / \mathrm{A}_{\mathrm{i}, \mathrm{t}-\mathrm{1}}
$$

Em que,

$\mathrm{TA}_{\mathrm{it}}=$ accruals totais da empresa i no período t;

$\Delta \mathrm{AC}_{\mathrm{i}, \mathrm{t}}=$ variação do ativo circulante da empresa i no período $\mathrm{t}-1$ para o período $\mathrm{t}$;

$\triangle$ Disp $_{\mathrm{i}, \mathrm{t}}=$ variação das disponibilidades da empresa i no período t-1 para o período $\mathrm{t}$;

$\Delta \mathrm{PC}_{\mathrm{i}, \mathrm{t}}=$ variação do passivo circulante da empresa i no período t-1 para o período t;

$\Delta \operatorname{Div}_{\mathrm{i}, \mathrm{t}}=$ variação das dividas de curto prazo da empresa i no período t-1 para o período t;

$\mathrm{DDA}_{\mathrm{i}, \mathrm{t}}=$ valor de depreciação e amortização da empresa i no período t;

$\mathrm{A}_{\mathrm{i}, \mathrm{t}-1}=$ total do ativo da empresa i no período $\mathrm{t}-1$.

Para mensurar os accruals não discricionários, e por conseguinte estimar os discricionários, segundo Martinez (2001), Paulo (2007) e Baptista (2008) o modelo Jones Modificado (Dechow et al., 1995) é o mais utilizado em pesquisas de âmbito internacional. Este trabalho apoia-se neste modelo, detalhado a seguir:

$$
\begin{aligned}
& \mathrm{TA}_{\mathrm{i}, \mathrm{t}}=\beta 1\left(1 / \mathrm{Ai}_{\mathrm{t},-\mathrm{l}}\right)+\beta 2\left(\Delta \text { Receitas }_{\mathrm{i}, \mathrm{t}}-\Delta \mathrm{CR}_{\mathrm{i}, \mathrm{t}} / \mathrm{A}_{\mathrm{i}, \mathrm{t}-\mathrm{l}}\right)+\beta 3\left(\mathrm{PPE}_{\mathrm{i}, \mathrm{t}} / \mathrm{A}_{\mathrm{i}, \mathrm{t}-\mathrm{l}}\right)+\varepsilon_{\mathrm{i}, \mathrm{t}} \\
& N A_{i, t}=\beta 1\left(1 / A_{i, t-1}\right)+\beta 2\left(\Delta \text { Receitas }_{i, t}-\Delta \mathrm{CR}_{\mathrm{i}, \mathrm{t}} / \mathrm{A}_{\mathrm{i}, \mathrm{t}-\mathrm{l}}\right)+\beta 3\left(\mathrm{PPE}_{\mathrm{i}, \mathrm{t}} / \mathrm{A}_{\mathrm{i}, \mathrm{t}-\mathrm{l}}\right) \\
& D A_{i, t}=\mathrm{TA}_{\mathrm{i}, \mathrm{t}}-\mathrm{NDA}_{\mathrm{i}, \mathrm{t}}
\end{aligned}
$$

Em que,

$\mathrm{TA}_{\mathrm{i}, \mathrm{t}}=$ accruals totais da empresa i no período $\mathrm{t}$;

$\mathrm{NDA}_{\mathrm{i}, \mathrm{t}}=$ accruals não discricionários da empresa i no período t;

$\mathrm{DA}_{\mathrm{i}, \mathrm{t}}=$ accruals discricionários da empresa i no período t;

$A i_{t-1}=$ total do ativo da empresa i no período $\mathrm{t}-1$; 
$\Delta$ Receitas $_{\mathrm{i}, \mathrm{t}}=$ variação das receitas líquidas da empresa i no período t-1 para o período t; $\Delta C \mathrm{R}_{\mathrm{i}, \mathrm{t}}$ v variação de contas a receber da empresa i no período $\mathrm{t}-1$ para o período $\mathrm{t}$; $\mathrm{PPE}_{\mathrm{i}, \mathrm{t}}=$ ativo imobilizado (bruto) somado ao intangível da empresa i no período t; $\beta 1 ; \beta 2 ; \beta 3=$ Coeficientes estimados pela regressão;

$\varepsilon_{\mathrm{i}, \mathrm{t}}=$ Erro da regressão da empresa i no período $\mathrm{t}$ (proxy para gerenciamento de resultados).

Com este modelo observa-se que mudanças no nível das receitas impactam no capital de giro da empresa (contas a receber, contas a pagar e estoques); e também, mudanças no ativo imobilizado (bruto) e intangível estão refletidas em despesas com depreciação, e amortização. Desta forma, o modelo supõe que as variações nos accruals totais podem ser explicadas pelas variações na receita deduzidas do contas a receber, bem como do ativo imobilizado e intangível da empresa. Cabe ressaltar que a inclusão do intangível no PPE se dá pelas seguintes razões: primeiramente devido às empresas de utilidade pública terem sido orientadas, com base no Interpretações e Orientações Técnicas e Contábeis (ICPC) 01 (R1) em 2009, a reclassificarem seus ativos Imobilizados (relacionados aos contratos de concessão) em Intangível, fazendo com que parte da captação dos accruals ficasse prejudicada no caso da não inserção desta variável no cálculo; e também pelo fato de que o Intangível segue a lógica do Imobilizado no que tange à discricionariedade na contabilização, pois assim como os accruals podem ser manipulados por meio da depreciação, é possível também manipulá-los por meio da amortização; por estes motivos, e considerando que o DDA inclui amortização no cálculo, foi considerado o Intangível no PPE. O erro do modelo representa a proxy de GR, entendido como uma variação não esperada de accruals (Jones, 1991).

Inicialmente então por meio da Equação 2.1 é possível levantar os accruals totais, e em seguida através dos coeficientes $\beta 1, \beta 2$ e $\beta 3$ da Equação 2.2 é possível estimar os accruals não discricionários, medidos pelo resíduo da regressão. Quanto mais distante de zero (positiva ou negativamente) o resíduo estiver, maior o Gerenciamento de Resultados.

Malmendier e Tate (2005) propõem três medidas para excesso de confiança e otimismo, denominadas por eles como: Holder 67, Longholder e Net Buyer. As duas primeiras utilizam o tempo referente ao exercício de opções de ações (stock options) em posse dos CEOs, e a terceira usa a aquisição líquida de ações da própria companhia, também analisando a carteira pessoal dos CEOs. No Brasil, nem todas empresas dispõem em seu estatuto social a remuneração baseada no exercício de opção de ações, o que poderia reduzir a amostra caso fosse necessário o levantamento destes dados. Por essa razão, o modelo escolhido para mensurar os vieses foi o Net Buyer.

Segundo esta lógica, se o gestor é excessivamente confiante e otimista o número de ações adquiridas da empresa em que atua é maior em comparação com o número de ações vendidas num determinado período, sendo chamado então de'comprador habitual', devido à sua compra líquida ser positiva. Assim, o gestor aumenta seu risco idiossincrático por não estar diversificando sua carteira. É razoável supor que esta decisão se afasta dos parâmetros de racionalidade, devido ao fato de o gestor acreditar que a empresa é suficientemente propensa a resultados favoráveis (positivos) subestimando os riscos e superestimando a capacidade de gestão, levando assim à caracterização de um comportamento tanto excessivamente confiante quanto otimista. Esta interpretação está em conformidade com Malmendier e Tate (2008) que consideram que suas métricas para Overconfidence consideram o excesso de confiança propriamente dito e também otimismo. Os dados referentes à compra e venda de ações dos CEOs não são divulgados separadamente, estando evidenciados no montante de transações realizadas por estes e os demais di- 
retores. Hochberg (2011) argumenta que os demais diretores não são considerados independentes dos CEOs e que estes exercem influência sobre suas decisões, dado que seu sucesso está atrelado ao do CEO. Por estas razões os dados levantados dizem respeito às transações realizadas pelos directors (diretores).

Entretanto, apenas o valor da compra líquida (em número de ações) pode não representar tão bem o nível do viés comportamental, pois ainda que uma empresa $X$ apresente um valor de compra líquida maior que a empresa $Y$, pode ser que o valor da ação desta empresa seja superior ao da outra. Tal fato faz com que o valor monetário depositado pela empresa $Y$ seja superior ao da empresa $X$, e consequentemente os CEOs dela estariam mais confiantes e otimistas, se arriscando mais que os da outra. Para se aproximar do valor depositado pelos diretores em suas transações, foi coletado o valor de fechamento do ano das respectivas ações das empresas. O cálculo da proxy dos vieses de excesso de confiança e otimismo foi feito, então, pela equação a seguir:

$$
\mathrm{ECO}_{\mathrm{i}, \mathrm{t}}=\left(\text { Compra }_{\mathrm{i}, \mathrm{t}} \text { Venda }_{\mathrm{i}, \mathrm{t}} \mathrm{x} \text { Preço da ação } \mathrm{i}_{\mathrm{i}, \mathrm{t}}\right.
$$

Em que,

$\mathrm{ECO}_{\mathrm{i}, \mathrm{t}}=$ índice de excesso de confiança e otimismo da empresa i no período t;

Comprai, $t=$ número de ações da própria empresa compradas pelos directors da empresa i no período t;

Vendai, $\mathrm{t}=$ número de ações da própria empresa vendidas pelos directors da empresa i no período $\mathrm{t}$;

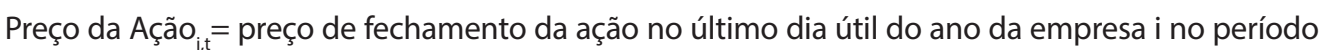
t-1 para o período t.

É importante salientar que, para compor a compra líquida (Compra - Venda) foram consideradas apenas aquelas empresas que realizaram transação em pelo menos 1 dos anos que abrange o estudo. Isto para que não entrassem na amostra empresas que tenham políticas restritivas quanto à aquisição de ações pelos CEOs, e assim que estariam sendo caracterizadas como não excessivamente confiantes e otimistas (dado que nestes casos a compra líquida seria igual a zero) e possivelmente enviesando o resultado da pesquisa.

Dado isto, pretende-se testar a seguinte hipótese:

H1: Há relação significativa e positiva entre o gerenciamento de resultados e os vieses de excesso de confiança e otimismo.

Na sequência são apresentadas as demais variáveis que se acredita influenciar o gerenciamento de resultados.

Foram inseridas no modelo as variáveis Crescimento, Desempenho, Alavancagem, Tamanho e Setor da economia por serem as variáveis que vêm sendo mais utilizadas por autores nacionais e internacionais (Dechow et al. 1995; Kothari, Leone \& Wasley, 2005; Morsfield \& Tan, 2006; Baptista, 2008; Hochberg, 2011; Barros, 2012; Bouwman, 2014; Consoni, 2014; Kermani et al. 2014; Melo, 2015) como condicionantes do gerenciamento de resultados contábeis.

O Figura 1 demonstra um resumo das variáveis da pesquisa, independente e de controle, inseridas no modelo. 
Figura 1 - Variáveis condicionantes para gerenciamento de resultados

\begin{tabular}{|c|c|c|c|}
\hline Variáveis e (Siglas) & Operacionalização & Efeito esperado & Referências \\
\hline $\begin{array}{l}\text { Excesso de Confiança } \\
\text { e Otimismo (ECO) }\end{array}$ & $\begin{array}{c}\text { Produto da compra líquida (quantidade } \\
\text { comprada menos vendida) de açōes da } \\
\text { própria empresa pelos diretores e o preço } \\
\text { de fechamento do ano das respectivas } \\
\text { ações. }\end{array}$ & $(+)$ & $\begin{array}{c}\text { Li \& Hung (2013), Bouwman (2014), Hsieh, } \\
\text { Bedard \& Johnstone (2014) e Kermani, Kargar e } \\
\text { Zarei (2014). }\end{array}$ \\
\hline Crescimento (CRES) & $\begin{array}{c}\text { Variação na receita operacional líquida } \\
\text { da empresa i entre } t-1 \text { e } t \text {, dividida pela } \\
\text { receita operacional líquida da empresa } \\
\text { i em } t \text { - } 1\end{array}$ & $(+)$ & $\begin{array}{c}\text { Baptista (2008), Hochberg (2011), Li \& Hung } \\
\text { (2013) e Sincerre, Sampaio, Famá \& Santos } \\
\text { (2016). }\end{array}$ \\
\hline Desempenho (ROA) & $\begin{array}{c}\text { Retorno sobre os ativos (ROA) calculado } \\
\text { como a razão entre o lucro líquido e os } \\
\text { ativos totais. }\end{array}$ & $(+)$ & $\begin{array}{l}\text { Kothari, Leone e Wasley (2005), Baptista (2008), } \\
\text { Bouwman (2014), Kermani et al. (2014) e Sincerre } \\
\text { et al. (2016). }\end{array}$ \\
\hline Alavancagem (ALAV) & $\begin{array}{l}\text { Alavancagem calculada como a razão entre } \\
\text { o capital de terceiros e os ativos totais. }\end{array}$ & $(+)$ & $\begin{array}{l}\text { Morsfield e Tan (2006), Baptista (2008), Hochberg } \\
\text { (2011), Li \& Hung (2013), Kermani et al. (2014) e } \\
\text { Sincerre et al. (2016). }\end{array}$ \\
\hline Tamanho (TAM) & $\begin{array}{l}\text { Logaritmo natural da receita líquida da } \\
\text { empresa i no trimestre } t \text { (em milhões de } \\
\text { reais). }\end{array}$ & $(+)$ & $\begin{array}{l}\text { Baptista (2008), Castro Junior, Conceição \& } \\
\text { Santos (2011) e Sincerre et al. (2016). }\end{array}$ \\
\hline $\begin{array}{l}\text { Setor da economia } \\
\text { (SETOR) }\end{array}$ & Dummy & Incerto & $\begin{array}{l}\text { Barros (2012), Consoni (2014) e Kermani et al. } \\
\text { (2014). }\end{array}$ \\
\hline
\end{tabular}

Fonte: Dados da pesquisa.

Estas variáveis foram inseridas no modelo para explicar os níveis de accruals discricionários obtidos pelas empresas (variável dependente), ou seja, os condicionantes para a prática de gerenciamento de resultados contábeis. O modelo e as técnicas aplicadas estão descritas a seguir.

\subsection{Modelo Econométrico e Técnicas de Análise de Dados}

De posse dos dados, é realizada sua tabulação para o cálculo das estatísticas descritivas das variáveis da pesquisa, procede-se à análise multivariada com a aplicação da regressão, a fim de verificar a relação entre a variável dependente e as demais variáveis explicativas.

Foram testados, para o modelo, os seguintes pressupostos: (1) ausência de autocorrelação (Teste de Durbin-Watson), (2) ausência de heterocedasticidade (Teste de White), (3) normalidade dos resíduos (Teste de Shapiro-Wilk) e (4) ausência de multicolineariedade (Fator de Inflação da Variância - VIF). O modelo pode ser descrito através da seguinte equação:

$$
\mathrm{GR}_{i, t}=\beta 0+\beta 1 \mathrm{ECO}_{i, t}+\beta 2 \mathrm{CRES}_{i, t}+\beta 3 \mathrm{ROA}_{\mathrm{i}, \mathrm{t}}+\beta 4 \mathrm{ALAV}_{\mathrm{i}, \mathrm{t}}+\beta 5 \mathrm{TAM}_{\mathrm{i}, \mathrm{t}}+\beta 6 \mathrm{SETOR}_{\mathrm{i}, \mathrm{t}}+\varepsilon_{\mathrm{i}, \mathrm{t}}
$$

Em que,

$\mathrm{GR}_{\mathrm{i}, \mathrm{t}}=$ nível de gerenciamento de resultados da empresa i no período $\mathrm{t}$;

$\mathrm{ECO}_{\mathrm{i}, \mathrm{t}}$ = índice de excesso de confiança e otimismo da empresa i no período t;

$\mathrm{CRES}_{\mathrm{i}, \mathrm{t}}=$ medida de crescimento da empresa i no período $\mathrm{t}$;

$\mathrm{ROA}_{\mathrm{i}, \mathrm{t}}=$ retorno sobre ativos da empresa i no período t;

$A L A V_{i, t}=$ alavancagem da empresa i no período $t ;$

$\mathrm{TAM}_{\mathrm{i}, \mathrm{t}}=$ tamanho da empresa i no período $\mathrm{t}$;

$\mathrm{SETOR}_{\mathrm{i}, \mathrm{t}}=$ setor da empresa i no período t;

$\beta 0 ; \beta 1 ; \beta 2 ; \beta 3 ; \beta 4 ; \beta 5 ; \beta 6=$ coeficientes do modelo de regressão;

$\varepsilon_{\mathrm{i}, \mathrm{t}}=$ erro da regressão da empresa i no período t.

Por trabalhar com dados de diversas empresas 'i' observadas em diferentes momentos no tempo ' $\mathrm{t}$ ' (tempo), a análise varia em espaço e tempo constituindo um Painel de observações (Gujarati, 2006). Tanto a estimação dos coeficientes do modelo de Gerenciamento de Resultados, quanto a Equação 2.6, 
foram realizadas por meio da regressão de Dados em Painel Balanceado, pois as empresas do início do período são as mesmas do final do período. Ao adotar esse método deve-se realizar os diagnósticos de painel, que englobam os testes de Chow, Breusch-Pagan e Hausman para identificar quais dos seguintes modelos, Pooled Ordinary Least Squares (Pooled OLS), Efeitos Fixos ou Efeitos Aleatórios, são adequado para este estudo. Os testes estatísticos foram realizados com auxílio do software STATA ${ }^{\oplus}$ versão 12.1.

\section{APRESENTAÇÃO E DISCUSSÃO DOS RESULTADOS} 4.1 Gerenciamento de Resultados - Accruals Discricionários (AD)

Para apurar esta proxy foi utilizado o modelo de Jones Modificado (Dechow et al., 1995), apresentado na seção 2.2 deste trabalho. Por meio deste modelo, é possível apurar os $A D$, representados pelo erro da regressão [equação 2.3 ].

Em relação aos testes dos pressupostos do modelo de regressão, verificou-se que o teste de Durbin-Watson para autocorrelação não rejeitou a hipótese nula de ausência de correlação (Durbin-Watson $\mathrm{d}$-statistic $[4,680]=1.877022)$. Entretanto, o teste de Breusch-Pagan para heterocedasticidade apresentou significância (Prob>chi ${ }^{2}=0.0000$ ) rejeitando a hipótese de que os dados são homocedásticos, levando assim a um novo processamento da regressão, agora com erros robustos. Na tabela 1 são apresentados os testes estatísticos do modelo Jones Modificado (Dechow et al., 1995):

Tabela 1 - Resultados da regressão (pooled OLS) - modelo Jones Modificado (1995)

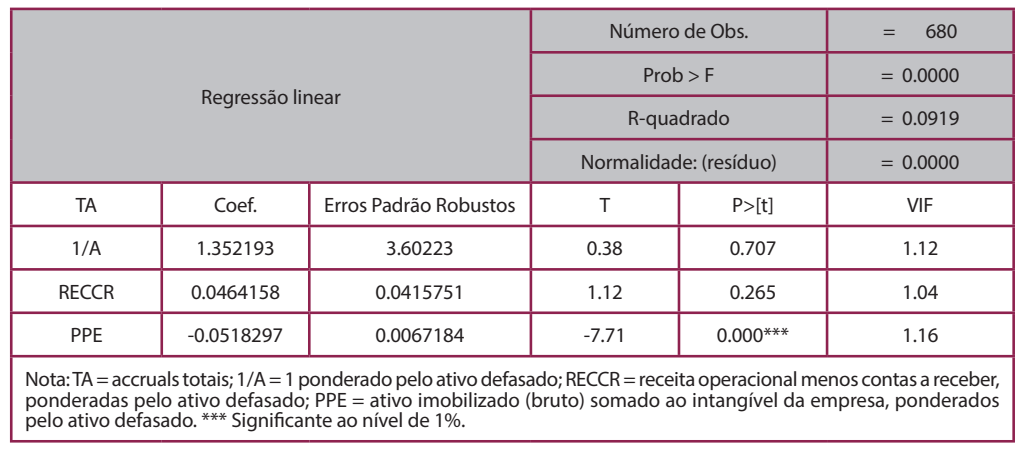

Fonte: elaborado pelo autor

A Tabela 1 indica por meio do teste $F$ que o modelo pooled é significativo (Prob $>F=0.0000$ ) validando assim sua aplicação. É possível verificar um R2 de 0.0919, e que apenas a variável PPE explica as variações de TA (ao nível de significância de 1\%), com coeficiente de 0.0518297 negativo. As demais não apresentaram significância, sugerindo que a variabilidade de A e RECCR não são explicativas para as variações de TA. O VIF (Fator de Inflação de Variância) mostra que o modelo não possui problemas de multicolinearidade na especificação, dado que os valores estão todos próximos de 1. Adicionalmente, os resultados sugerem a não normalidade dos resíduos (Prob>chi2 =0.0000), entretanto dado o número de observações (680 observações) a literatura aceita que eles estão próximos de uma distribuição normal (Wooldridge, 2012).

De posse dos resultados e do erro da regressão, que representa o gerenciamento de resultados por meio dos accruals discricionários, partiu-se então para a aplicação do modelo que relaciona o gerenciamento com o excesso de confiança e demais variáveis de controle. 


\subsection{Accruals Discricionários vs Excesso de Confiança e Otimismo}

Para testar a relação entre gerenciamento de resultados e o excesso de confiança e otimismo foi utilizada a regressão descrita na equação [2.6]. Espera-se, a priori, verificar uma relação direta entre o gerenciamento de resultados e a gestão excessivamente confiante e otimista. Inicialmente a equação foi estimada por pooled OLS, em seguida foram realizados os testes de Breusch-Pagan/Cook-Weisberg para heterocedasticidade e o teste de Durbin-Watson para autocorrelação. O teste de heterocedasticidade rejeitou a hipótese nula, apresentando dados não homocedásticos, e então foi refeito com a utilização de erros padrão robustos. Um outro ponto importante a destacar, é o Fator de Inflação da Variância (VIF) que apresentou valores superiores a 10 excedendo o permitido pela literatura, e para solucionar este problema foi eliminado da regressão a variável que apresentou o maior VIF, CCIC no caso (VIF = 28.95). Após este procedimento as variáveis não apresentaram mais problemas de multicolinearidade. Também foi necessário realizar os testes de diagnósticos de painel, que têm a finalidade de identificar qual modelo, entre pooled, efeitos fixos ou efeitos aleatórios, melhor representa a relação entre o gerenciamento de resultados e as demais variáveis independente e de controle. Na sequência, a Tabela 2 evidencia os resultados dos testes de diagnóstico de painel.

Tabela 2 - Diagnóstico do modelo [2.6] em painel

\begin{tabular}{|c|c|}
\hline Testes & $\mathrm{p}$-valor \\
\hline Teste de Chow & 0.356519 \\
\hline Teste de Breusch-Pagan & 0.769788 \\
\hline Teste de Hausman & 0.334168 \\
\hline
\end{tabular}

Fonte: elaborado pelo autor

O teste de Chow indica que o modelo pooled OLS (MQO) é mais apropriado que o modelo de efeitos fixos, e este fica sendo o modelo utilizado nesta pesquisa. Na Tabela 3 são apresentados os resultados da regressão pelo modelo pooled OLS com erros robustos à heterocedasticidade.

Tabela 3 - Resultados da regressão (pooled OLS), erros padrão robustos - modelo [2.6], DA vs ECO

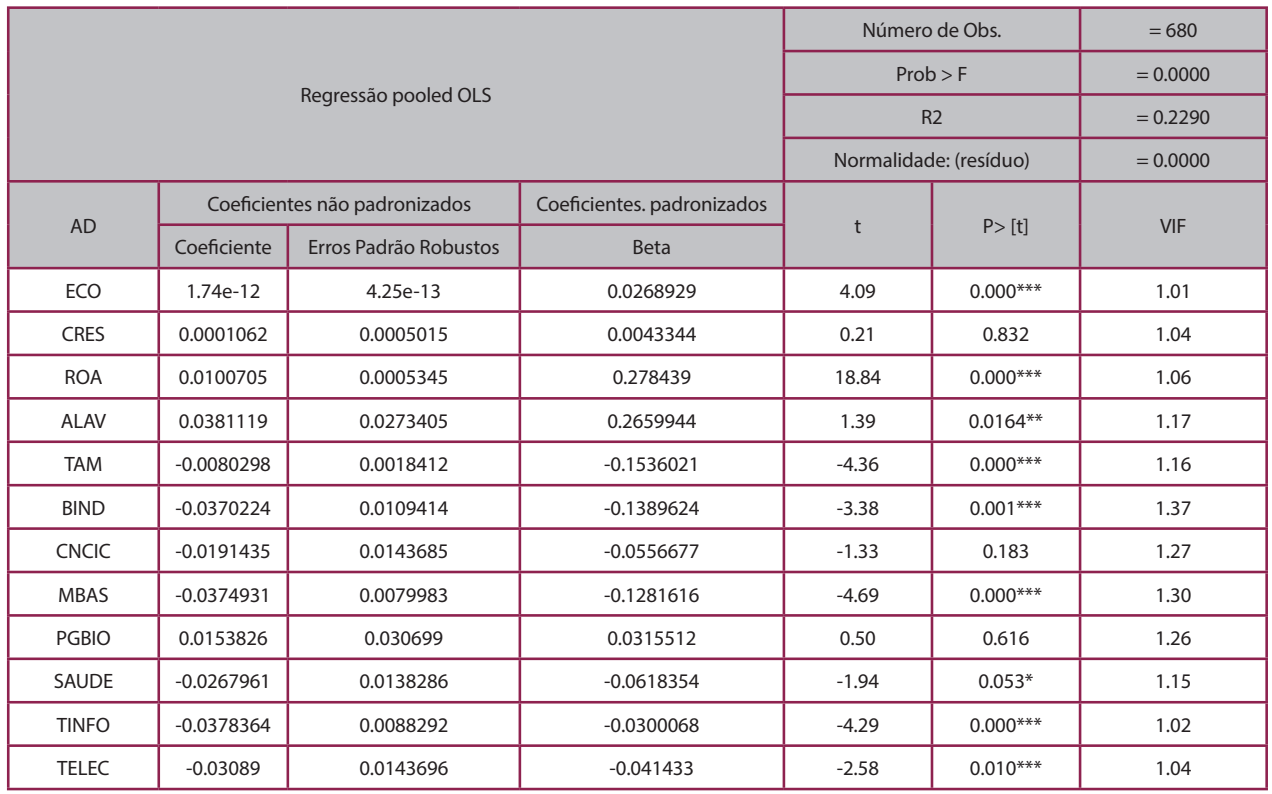




\begin{tabular}{|c|c|c|c|c|c|c|}
\hline UPUBL & -0.0387509 & 0.0082392 & -0.1020295 & -4.70 & $0.000^{* * *}$ & 1.22 \\
\hline _cons & 0.1185159 & 0.0257053 & 0.0000 & 4.61 & $0.000^{* * *}$ & \\
\hline
\end{tabular}

Nota: $A D=$ accruals discricionários; $E C O$ = excesso de confiança e otimismo; $C R E S=$ crescimento da receita operacional líquida; $R O A=$ rentabilidade do ativo; ALAV = medida de alavancagem (endividamento); TAM = logaritmo natural da receita operacional; $B I N D=$ variável dummy para setor de Bens Industriais; $C N C I C$ = variável dummy para setor de Consumo Não-Ciclico; MBAS = variável dummy para setor de Materiais Básicos; PGBIO = variável dummy para setor de Petróleo, Gás e Biocombustiveis; SAUDE = variável dummy para setor de Saúde; TINFO = variável dummy para Pública.***,*** e $^{*}$ Significante ao nível de $1 \%, 5 \%$ e $10 \%$.

Fonte: elaborado pelo autor

O teste apresentou significância a nível de $1 \%$ na estatística F, e um poder explicativo (R2) de 0,2290, ou seja, as variáveis do modelo foram capazes de explicar aproximadamente $22,9 \%$ das variações dos accruals discricionários. Este resultado está próximo dos achados de pesquisas internacionais como: Li e Hung (2013), com 0,096; Bouwman (2014) com 0,16; Hsieh et al. (2014) com 0,195 e; Kermani et al. (2014) com 0,156. Também é possível verificar na Tabela 3 que o modelo não apresentou problemas de multicolinearidade, entretanto o teste de normalidade dos resíduos apresentou significância, mas o Teorema do Limite Central aponta que os estimadores de pooled OLS satisfazem a normalidade assintótica, ou seja, dado que esta pesquisa conta com um grande número de observações (680) eles estão próximos de uma distribuição normal (WOOLDRIDGE, 2012).

A variável independente excesso de confiança e otimismo foi significativa ( $p$-valor $=0.000$ ). Esse resultado está em consonância com a hipótese teórica do presente estudo, de que o viés de excesso de confiança e otimismo está relacionado com o gerenciamento de resultados. $\mathrm{O}$ trabalho de Bouwman (2014) apresentou significância estatística nesta relação, mas ao contrário deste estudo, o sinal encontrado foi negativo, sugerindo uma relação inversa entre as variáveis independente e dependente. Uma possível explicação para a divergência de sinais encontrados é o fato de o autor ter trabalhado com uma modalidade específica de gerenciamento de resultados, o Income Smoothing (Suavização de Resultados), enquanto que este estudo trabalha com o gerenciamento no sentido amplo.

Já os resultados de Hsieh et al. (2014) e Li \& Hung (2013) estão de pleno acordo com os achados desta pesquisa, inclusive com a coincidência do sinal encontrado (positivo). Isto sugere que CEOs mais excessivamente confiantes e otimistas gerenciam mais seus resultados. Hsieh et al. (2014) sugerem que esse resultado se dá porque gestores são sensíveis ao comportamento excessivamente confiante ou 'narcisista', especialmente o CEO, mas possivelmente outros membros da gestão que estejam em posição de gerenciar os resultados. Li \& Hung (2013) indicam que além da teoria da agência, assimetria de informação, a hipótese de fluxo de caixa livre, dentre outros, o aspecto comportamental, especificamente o excesso de confiança, ajuda a aumentar a compreensão de porque os gestores têm incentivos para manipular os resultados da empresa.

Com relação à variável crescimento, ela não apresentou significância estatística, indicando que a mesma não guarda relação com gerenciamento de resultados. Por outro lado, a variável alavancagem apresentou sinal positivo e significante, ou seja, verifica-se que empresas com maior grau de alavancagem, gerenciam mais seus resultados. Rezende e Nakao (2012) argumentam que quanto mais endividadas as empresas estiverem, maior é o incentivo para gerenciar seus resultados, possivelmente para obtenção ou renovação de empréstimos ou financiamentos, ou ainda para evitar cláusulas restritivas baseadas em dados contábeis.

Já o ROA, variável utilizada para medir o desempenho, apresentou significância estatística (p-valor $=0.000$ ) e sinal positivo, conforme era esperado pela construção teórica da pesquisa, e semelhante aos estudos de Baptista (2008), Castro (2008), Barros (2012) e Sincerre et al. (2016). Isto sugere que empresas com maiores retornos gerenciam mais seus resultados, algo de se esperar dado que eles podem estar 
gerenciando seus resultados para reportar maior rentabilidade com o interesse de influenciar a percepção de mercado (investidores e credores).

A variável tamanho, medida pelo logaritmo natural do ativo, apresentou relação negativa e significativa, sugerindo que as empresas de maior porte gerenciam menos seus resultados. Esta evidencia está de acordo com o esperado, e Gu, Lee e Rosett (2005) defendem que empresas maiores operam em estado mais sólido e tendem a ser mais diversificadas, apresentando menor variabilidade operacional e consequentemente menor variabilidade dos accruals discricionários. Este resultado apresenta semelhanças com os achados de Morsfield e Tan (2006), Castro (2008), Barros (2012), Li \& Hung (2013), Bouwman (2014) e Sincerre et al. (2016).

Por fim, foram analisadas as classificações setoriais segundo a B3, e sua relação com o gerenciamento de resultados. Dos oito setores que constaram nesta análise, apenas três não apresentaram significância, sendo eles o Consumo não-Cíclico, o de Petróleo, Gás e Biocombustíveis e o de Saúde. Dentre os significantes, pode-se verificar que o setor mais propenso a prática de gerenciamento é o de Bens Industriais que apresentou um coeficiente padronizado $(\beta)$ de -0.1389624 , seguido do setores de Materiais Básicos ( $\beta=-0.1281616)$, Utilidade Pública ( $\beta=-0.1020295)$, Telecomunicações $(\beta=-0.0414433)$ e Tecnologia da Informação ( $\beta=-0.0300068$ ). Esta análise serviu para verificar a significância de agrupamentos setoriais como fatores explicativos para as variações dos accruals discricionários.

Vale ressaltar que dada a grande dispersão na variável ECO (mínimo -4.34e+10 e máximo 2.35e+09), foi considerado adicionalmente o coeficiente padronizado beta, gerado com a utilização do SPSS. Tal coeficiente equivale a utilizar a regressão com todas as variáveis normalizadas de acordo com o escore $Z$ da variável normal. Ao realizar esta transformação, as variáveis passam a possuir, de acordo com Gujarati (2006), média zero e desvio padrão 1, e em consequência não importa em que unidades se expressem o regressando e os regressores. Desta forma, o efeito escala da variável ECO deixa de existir. A vantagem de se utilizar este procedimento de padronização dos regressores é que todos ficam na mesma base, e assim pode-se compara-los diretamente, ou seja, "se o coeficiente de um regressor padronizado for maior que o de outro regressor padronizado que conste do mesmo modelo, então ele contribui mais em relação à explicação do regressando do que o segundo" (GUJARATI, 2006, p. 240). A equação com os coeficientes beta mantém a significância, mas deve ser interpretada em unidades de desvios padrão. Assim, considerando os resultados apresentados na Tabela 3, a equação [2.6] com os coeficientes padronizados Beta pode ser reescrita da seguinte forma:

$$
\begin{aligned}
& \mathrm{GR}_{\mathrm{i}, \mathrm{t}}=0,0268 \mathrm{ECO}_{\mathrm{i}, \mathrm{t}}+0,2784 \mathrm{ROA}_{\mathrm{i}, \mathrm{t}}+0,2660 \mathrm{ALAV}_{\mathrm{i}, \mathrm{t}}-0,1536 \mathrm{TAM}_{\mathrm{i}, \mathrm{t}}-0,1390 \mathrm{BIND}_{\mathrm{i}, \mathrm{t}}-0,1282 \mathrm{MBA}- \\
& \mathrm{S}_{\mathrm{i}, \mathrm{t}}-0,0618 \mathrm{SAUDE}_{\mathrm{i}, \mathrm{t}}-0,0300 \mathrm{TINFO}_{\mathrm{i}, \mathrm{t}}-0,0414 \mathrm{TELEC}_{\mathrm{i}, \mathrm{t}}-0,1020 \text { UPUBL }_{\mathrm{i}, \mathrm{t}}+\varepsilon_{\mathrm{i}, \mathrm{t}}
\end{aligned}
$$

\section{CONSIDERAÇÕES FINAIS}

A decisão de comprar e vender ações envolve riscos associados a diversos fatores, os quais podem trazer ao investidor ganhos altos, mas altas perdas também. Neste sentido, esta pesquisa apurou a compra líquida dos CEOs, ou seja, suas compras menos vendas de ações das companhias em que atuam, partindo do pressuposto que quando o CEO (ou qualquer investidor) opera maior compra, em valores reais, há superestimação da capacidade gerencial de gerar resultados positivos ou subestimação dos riscos da ocorrência de eventos que levem a resultados negativos (excesso de confiança e otimismo, 
respectivamente). Esta é a lógica por trás da métrica (net buyer) de Malmendier e Tate (2005), adaptada para o presente estudo, com a finalidade de caracterizar os vieses de excesso de confiança e otimismo.

Para investigar a influência destes vieses cognitivos sobre o gerenciamento de resultados, utilizou-se nesta pesquisa os accruals discricionários estimados a partir do modelo Jones Modificado (Dechow et al., 1995). Adicionalmente foram testadas como variáveis de controle: o crescimento, desempenho, alavancagem, tamanho e setor de atuação das empresas da amostra como fatores explicativos para o gerenciamento de resultados.

$\mathrm{Na}$ análise realizada constatou-se que o excesso de confiança e o otimismo estão relacionados positiva e significativamente com o gerenciamento de resultados, fato que confirma a hipótese teórica deste estudo e segue em conformidade com outros estudos (Malmendier \& Tate, 2008; Li \& Hung, 2013; Hsieh et al., 2014; Hribar \& Yang, 2015). Estes resultados sugerem que CEOs mais excessivamente confiantes e otimistas, gerenciam mais os resultados da empresa, ou seja, infere-se que gestores, por superestimar suas capacidades e habilidades (excessivamente confiantes), e subestimando a probabilidade de eventos desfavoráveis (otimistas) gerenciam mais resultados por projetar que as empresas estão subavaliadas, de forma a apresentar resultados futuros positivos compatíveis com suas crenças particulares. Estes achados contribuem para a literatura da área ao trazer para âmbito nacional a influência destes vieses comportamentais sobre decisões gerenciais e com o mercado no sentido de ressaltar para os investidores e demais stakeholders a preocupação com fatores comportamentais que podem enviesar os resultados reportados pelas empresas.

Além disso, verificou-se significância estatística na relação das seguintes variáveis o com gerenciamento de resultados: Desempenho, Alavancagem, Tamanho e os setores de Bens Industriais, Materiais Básicos, Saúde, Tecnologia da Informação, Telecomunicações e Utilidade Pública. Tais variáveis foram utilizadas para controlar fatores exógenos à relação principal investigada no trabalho, e apesar de não serem o objeto de discussão neste trabalho, a sua relevância na explicação do gerenciamento de resultados suscita a realização de estudos futuros que discutam e aprofundem tais relações.

Este trabalho contribui com reflexões tanto acadêmicas quanto no âmbito das decisões práticas. No tocante ao aspecto acadêmico, os resultados fornecem evidências que ajudam a levar em consideração a influência do comportamento do CEO sobre suas decisões financeiras, dado que ele pode estar enviesado e gerenciar resultados de forma discricionária para atender objetivos pessoais. Como contribuição teórica, tem-se que pesquisas demonstram que gestores têm motivações para agir com discricionariedade, então este estudo adiciona ao trabalhar fatores comportamentais influenciando essa tomada de decisão. Com relação às contribuições práticas, é importante verificar o excesso de confiança e otimismo nos CEOs, tanto no processo de recrutamento gerencial como monitoramento do mercado por parte dos investidores, referente as atitudes não apenas dos CEOs mas também outros membros da diretoria que estejam em posição de participar do gerenciamento de resultados, pois esta se justifica uma informação importante no processo de tomada de decisão de investimento.

A pesquisa realizada vai além dos estudos anteriores, pois aplica no mercado brasileiro uma proxy consistente para os vieses de excesso de confiança e otimismo, e chega a conclusões coerentes com a literatura internacional. Tais achados são importantes no sentido de apontar que mesmo em mercados menos eficientes e ambientes mais conturbados que o americano, a relação entre os vieses comportamentais e o gerenciamento de resultados se manteve.

Como diversos estudos desta natureza, este trabalho apresenta limitações, na amostra, coleta de dados e nas variáveis utilizadas. A estimação do excesso de confiança e otimismo dos CEOs foi feita utili- 
zando a métrica baseada em Malmendier e Tate (2005), que tem sido utilizada por outros pesquisadores, mas que é uma proxy para caracterizar os vieses comportamentais, e não há na literatura uma medida perfeita para medir esse comportamento. Sugere-se nesse sentido que estes vieses comportamentais sejam mensurados de outras formas para que sejam comparados os resultados. Uma sugestão pode ser, por exemplo, a consideração da formação curricular dos gestores. Ainda, a utilização de uma variável pode exigir que se descarte empresas por problemas em relação à utilização desta variável. Assim, não foi possível o teste para verificar se houve viés na seleção da amostra, uma vez que optou-se por descartar empresas em que não houve nenhuma transação com ações por parte dos gestores, dado que poderia haver restrições a este tipo de transação por parte da empresa, o que iria prejudicar a mensuração. Por sua vez, o gerenciamento de resultados também fora estimado por meio de uma proxy, ainda que usual na literatura da área, mas que também está sujeita a críticas.

Por fim recomenda-se verificar a influência de outros vieses comportamentais, não somente relacionados ao excesso de confiança e otimismo. De toda forma, as conclusões aqui verificadas contribuem para instigar novas pesquisas que aprofundem a influência de aspectos comportamentais sobre a tomada de decisão da gestão.

\section{REFERÊNCIAS}

Avelar, E. A., \& Santos, T. S. (2011) Gerenciamento de resultados contábeis: uma análise das pesquisas realizadas no Brasil entre os anos de 2000 e 2009. Revista de Contabilidade do Mestrado em Ciências Contábeis da UERJ, v. 15, n. 3, p. 19-33.

Baptista, E. M. B. (2008). Análise do perfil das empresas brasileiras segundo o nível de gerenciamento de resultados. 303 p. Tese de doutorado. Universidade Federal do Rio Grande do Sul, RS, Brasil.

Barros, C. M. E. (2012). Gerenciamento de Resultados contábeis e a qualidade de Governança Corporativa: Um estudo empírico em empresas brasileiras de capital aberto. 148 p. Dissertação de mestrado. Universidade Federal do Paraná, PR, Brasil.

Barros, L. A. D. C., \& Silveira, A. D. M. (2008). Excesso de Confiança, Otimismo Gerencial e os Determinantes da Estrutura de Capital. Revista Brasileira de Finanças, v.6, n.3.

Bouwman, C.H. S. (2014). Managerial optimism and earnings smoothing. Journal of Banking \& Finance, v. 41, p. 283-303. http://dx.doi.org/10.1016/j.jbankfin.2013.12.019.

Cabello, O. G., \& Pereira, C. A. (2015). Efeitos das práticas de tributação do lucro na effective tax rate (ETR): uma abordagem da teoria das escolhas contábeis. Advances in Scientific \& Applied Accounting, v. 8, n. 3. DOI: $10.14392 /$ ASAA.2015080305

Carpenter, V. L., \& Feroz, E. H. (2001). Institutional theory and accounting rule choice: an analysis of four US state governments' decisions to adopt generally accepted accounting principles. Accounting, organizations and society, v. 26, n. 7-8, p. 565-596. DOI: https://doi.org/10.1016/S0361-3682(00)00038-6.

Castro Junior, F. H. F., Conceição, P. M., \& Santos, D. A. (2011). A relação entre o nível voluntário de transparência e o custo de capital próprio das empresas brasileiras não-financeiras. REAd-Revista Eletrônica de Administração, v. 17, n. 3. DOI: http://dx.doi.org/10.1590/S1413-23112011000300002.

Castro Junior, F. H. F. C., \& Famá, R. (2010). As novas finanças e a teoria comportamental no contexto da tomada de decisão sobre investimentos. REGE Revista de Gestão, v. 9, n. 2.

Cavalheiro, E. A. (2015). Determinantes da estrutura de capital das micro e pequenas empresas: uma abordagem comportamental. 240 p. Tese de doutorado. Universidade Federal de Santa Maria, RS, Brasil.

Cole, V., Branson, J., \& Breesch, D. (2013). Determinants influencing the IFRS accounting policy choices of European listed companies. Available at SSRN 2217498. 
Collin, S. O. Y., Tagesson, T., Andersson, A., Cato, J., \& Hansson, K. (2009). Explaining the choice of accounting standards in municipal corporations: Positive accounting theory and institutional theory as competitive or concurrent theories. Critical perspectives on Accounting, v. 20, n. 2, p. 141-174. DOI: https:// doi.org/10.1016/j.cpa.2008.09.003.

Consoni, S. (2014). Divulgação voluntária e sua relação com gerenciamento de resultados contábeis: evidências no contexto da convergência às normas internacionais de contabilidade no Brasil. 141 p. Dissertação de mestrado. Universidade Federal do Paraná, PR, Brasil.

Dallabona, L. F., Martins, J. A. S., \& Klann, R. C. (2014). Utilização do gerenciamento de resultados para a redução de custos políticos: uma análise a partir da DVA. Contextus-Revista Contemporânea de Economia e Gestão, v. 12, n. 2, p. 91-115. DOI: http://dx.doi.org/10.19094/contextus.v12i2.32179.

Dechow, P. M., Sloan, R. G., \& Sweeney, A. P. (1995). Detecting earnings management. Accounting review, p. 193-225.

Fama, E. F. (1975). Multiperiod consumption-investment decisions. In: Stochastic Optimization Models in Finance. p. 389-400.

Famá, R., Cioffi, P. L., \& Coelho, P. A. R. (2008). Contexto das finanças comportamentais: anomalias e eficiência do mercado de capitais brasileiro. REGE Revista de Gestão, v. 15, n. 2, p. 65-78. DOI: http://dx.doi. org/10.5700/issn.2177-8736.rege.2008.36638.

Fávero, L. P., Belfiore, P., Silva, F. D., \& Chan, B. L. (2009). Análise de dados: modelagem multivariada para tomada de decisões. Rio de Janeiro: Elsevier.

Felleg, R., Moers, F., \& Renders, A. (2012). Investor reaction to higher earnings management incentives of overoptimistic ceos. The Accounting Review, v. 85, n. 6, p. 1951-1984.

Ferreira, C. F., \& Yu, A. S. O. (2003). Todos acima da média: excesso de confiança em profissionais de finanças. Revista de Administração da Universidade de São Paulo, v. 38, n. 2.

Ge, W., Matsumoto, D., \& Zhang, J. L. (2011). Do CFOs have style? An empirical investigation of the effect of individual CFOs on accounting practices. Contemporary Accounting Research, v. 28, n. 4, p. 1141-1179. DOI: https://doi.org/10.1111/j.1911-3846.2011.01097.x.

Gil, A. C. (2002). Como elaborar projetos de pesquisa. São Paulo, v. 5, n. 61, p. 16-17.

Gujarati, D. N. (2006). Econometria Básica. Rio de Janeiro: Elsevier.

Hackbarth, D. (2008). Managerial traits and capital structure decisions. Journal of Financial and Quantitative Analysis, v. 43, n. 4, p. 843-881. DOI: https://doi.org/10.1017/S002210900001437X.

Halfeld, M., \& Torres, F. F. L. (2001). Finanças comportamentais: a aplicações no contexto brasileiro. Revista de administração de empresas, v. 41, n. 2, p. 64-71. DOI: http://dx.doi.org/10.1590/S003475902001000200007.

Haller, A., \& Wehrfritz, M. (2013). The impact of national GAAP and accounting traditions on IFRS policy selection: evidence from Germany and the UK. Journal of International Accounting, Auditing and Taxation, v. 22, n. 1, p. 39-56. DOI: https://doi.org/10.1016/j.intaccaudtax.2013.02.003.

Healy, P. M., \&Wahlen, J. M. (1999). A review of the earnings management literature and its implications for standard setting. Accounting horizons, 13(4), 365-383. DOI: https://doi.org/10.2308/acch.1999.13.4.365.

Heaton, J. B. (2002). Managerial optimism and corporate finance. Financial management, p. 33-45. DOI: $10.2307 / 3666221$.

Hilary, G., \& Hsu, C. (2011). Endogenous overconfidence in managerial forecasts. Journal of Accounting and Economics, 51(3), 300-313. DOI: https://doi.org/10.1016/j.jacceco.2011.01.002.

Hochberg, Y. V. (2011). Venture capital and corporate governance in the newly public firm. Review of Finance, v. 16, n. 2, p. 429-480. DOl: http://dx.doi.org/10.2139/ssrn.474542.

Hribar, P., \&Yang, H. (2016). CEO overconfidence and management forecasting. Contemporary Accounting Research, v. 33, n. 1, p. 204-227. DOI: https://doi.org/10.1111/1911-3846.12144. 
Hsieh, T. S., Bedard, J. C., \& Johnstone, K. M. (2014). CEO overconfidence and earnings management during shifting regulatory regimes. Journal of Business Finance \& Accounting, v. 41, n. 9-10, p. 1243-1268. DOI: https://doi.org/10.1111/jbfa.12089.

Jaafar, A., \& Mcleay, S. (2007). Country effects and sector effects on the harmonization of accounting policy choice. Abacus, v. 43, n. 2, p. 156-189. DOI: https://doi.org/10.1111/j.1467-6281.2007.00224.x.

Jones, J. J. (1991). Earnings management during import relief investigations. Journal of accounting research, p. 193-228. DOI: 10.2307/2491047.

Kahneman, D., \& Tversky, A. (1974). Judgment under uncertainty: Heuristics and biases. Science, v. 185, n. 4157, p. 1124-1131.

(2013). Prospect theory: An analysis of decision under risk. In: Handbook of the fundamentals of financial decision making: Part I. p. 99-127. DOI: 10.2307/1914185.

Kermani, E., Kargar, E. F., \& Zarei, E. (2014). The Effect of Managerial Overconfidence on Profit Smoothing Evidence from Tehran Stock Exchange. Research Journal of Finance and Accounting. Research Journal of Finance and Accounting, v. 5, n. 9, p. 111-119.

Kimura, H. (2003). Aspectos comportamentais associados às reações do mercado de capitais. RAE-eletrônica, v. 2, n. 1, p. 1-14.

Kothari, S. P., Leone, A. J., \&Wasley, C. E. (2005). Performance matched discretionary accrual measures. Journal of accounting and economics, v. 39, n. 1, p. 163-197. DOl: https://doi.org/10.1016/j.jacceco.2004.11.002.

Li, I. ., \& Hung, J. H. (2013). The moderating effects of family control on the relation between managerial overconfidence and earnings management. Review of Pacific Basin Financial Markets and Policies, v. 16, n. 02, p. 1350010. DOI: https://doi.org/10.1142/S0219091513500100.

Malmendier, U., \& Tate, G. (2005). CEO overconfidence and corporate investment. The journal of finance, v. 60, n. 6, p. 2661-2700. DOl: https://doi.org/10.1111/j.1540-6261.2005.00813.x.

. (2008). Who makes acquisitions? CEO overconfidence and the market's reaction. Journal of financial Economics, v. 89, n. 1, p. 20-43. DOl: https://doi.org/10.1016/j.jfineco.2007.07.002.

Marconi, M. A., \& Lakatos, E. M. (1991). Metodologia científica. São Paulo: Atlas.

Markowitz, H. (1952). Portfolio selection. The journal of finance, v. 7, n. 1, p. 77-91. DOI: https://doi.org/10.1111/j.1540-6261.1952.tb01525.x.

. (1959). Portfolio Selection, Cowles Foundation Monograph No. 16. John Wiley, New York. S. Moss (1981). An Economic theory of Business Strategy, Halstead Press, New York. TH Naylor (1966). The theory of the firm: a comparison of marginal analysis and linear programming. Southern Economic Journal (January), 32, 263-74.

Martinez, A. L. (2001). Gerenciamento dos resultados contábeis: estudo empírico das companhias abertas brasileiras. 167 p. Tese de doutorado. Universidade de São Paulo, SP, Brasil.

. (2008). Detectando earnings management no Brasil: estimando os accruals discricionários. Revista Contabilidade \& Finanças-USP, v. 19, n. 46. DOI: http://dx.doi.org/10.1590/S1519-70772008000100002.

Martins, G. A. (2002). Divulgação de trabalho: considerações sobre os doze anos do caderno de estudos. Revista Contabilidade \& Finanças, v. 13, n. 30, p. 81-88. DOI: http://dx.doi.org/10.1590/S151970772002000300007.

Martins, V. G., Paulo, E., \& Monte, P. A. (2016). O gerenciamento de resultados contábeis exerce influência na acurácia da previsão de analistas no Brasil? Revista Universo Contábil, v. 12, n. 3, p. 73-90. DOI:10.4270/ RUC.2016322.

Mazzioni, S., Prigol, V., Moura, G. D., \& Klann, R. C. (2015). Influência da governança corporativa e da estrutura de capital no gerenciamento de resultados. Revista Contemporânea de Contabilidade, v. 12, n. 27, p. 61-86. DOI: https://doi.org/10.5007/2175-8069.2015v12n27p61. 
Melo, P. H. F. (2015). "Gerenciamento" dos resultados contábeis e o desempenho das ofertas publicas iniciais de ações de empresas brasileiras. 250 p. Dissertação de mestrado. Universidade Federal de Minas Gerais-UFMG, MG, Brasil.

Modigliani, F., \& Miller, M. H. (1958). The cost of capital, corporation finance and the theory of investment. The American economic review, v. 48, n. 3, p. 261-297.

Morsfield, S. G., \& Tan, C. E. (2006). Do venture capitalists influence the decision to manage earnings in initial public offerings?. The Accounting Review, v. 81, n. 5, p. 1119-1150.

Nardi, P. C. C., \& Nakao, S, H. (2009). Gerenciamento de resultados e a relação com o custo da dívida das empresas brasileiras abertas. Revista Contabilidade \& Finanças, v. 20, n. 51, p. 77-100. DOI: http://dx.doi. org/10.1590/S1519-70772009000300006.

Nobes, C., \& Perramon, J. (2013). Firm size and national profiles of IFRS policy choice. Australian accounting review, v. 23, n. 3, p. 208-215. DOI: https://doi.org/10.1111/j.1835-2561.2012.00203.x.

Paulo, E. (2007). Manipulação das informações contábeis: uma análise teórica e empírica sobre os modelos operacionais de detecção de gerenciamento de resultados. 269 p. Tese de doutorado. Universidade de São Paulo, SP, Brasil.

Pimenta, D. P., Borsato, J. M. L. S., \& Ribeiro, K. C. S. (2012). Um estudo sobre a influência das características sociodemográficas e do excesso de confiança nas decisões dos investidores, analistas e profissionais de investimento à luz das Finanças Comportamentais. REGE-Revista de Gestão, v. 19, n. 2, p. 263-280. DOI: http://dx.doi.org/10.5700/issn.2177-8736.rege.2012.49881.

Pinto, M. J. T., Martins, V. A., \& Silva, D. M. (2015). Escolhas contábeis: o caso brasileiro das propriedades para investimento. Revista Contabilidade \& Finanças-USP, v. 26, n. 69, p. 274-289. DOI: 10.1590/1808$057 \times 201512280$.

Reis, E. M., Lamounier, W. M., \& Bressan, V. G. F. (2015). Evitar Divulgar Perdas: Um Estudo Empírico do Gerenciamento de Resultados por Meio de Decisões Operacionais. Revista Contabilidade \& Finanças, v. 26, n. 69, p. 247-260. DOI: http://dx.doi.org/10.1590/1808-057x201501070.

Rezende, G. P., \& Nakao, S. H. (2012). Gerenciamento de resultados e a relação com o lucro tributável das empresas brasileiras de capital aberto. Revista Universo Contábil, v. 8, n. 1. DOI:10.4270/RUC.2012101

Roll, R. (1986). The hubris hypothesis of corporate takeovers. Journal of business, p. 197-216.

Schaefer, P. S., Williams, C. C., Goodie, A. S., \& Campbell, W. K. (2004). Overconfidence and the big five. Journal of research in Personality, v. 38, n. 5, p. 473-480. DOI: https://doi.org/10.1016/j.jrp.2003.09.010.

Schrand, C. M., \& Zechman, S. L. C. (2012). Executive overconfidence and the slippery slope to financial misreporting. Journal of Accounting and Economics, v. 53, n. 1-2, p. 311-329. DOI: https://doi.org/10.1016/j. jacceco.2011.09.001.

Sharpe, W. F. (1963). A simplified model for portfolio analysis. Management science, v. 9, n. 2, p. $277-293$. DOI: https://doi.org/10.1287/mnsc.9.2.277.

. (1964). Capital asset prices: A theory of market equilibrium under conditions of risk. The journal of finance, v. 19, n. 3, p. 425-442. DOI: https://doi.org/10.1111/j.1540-6261.1964.tb02865.x.

Silva, D. M., Martins, V. A., \& Lemes, S. (2016). Escolhas Contábeis: reflexões para a pesquisa. Revista Contemporânea de Contabilidade, v. 13, n. 29.

Silva, W. V., Piccoli, P. G. R., Cruz, J. A. W., \& Clemente, A. (2014). A eficiência do mercado de capitais brasileiros pela análise do efeito momento. Revista Economia \& Gestão, v. 14, n. 36, p. 113-137. DOI: 10.5752/P. 1984-6606.2014V14N36P113

Simon, H. (1957). Administrative behavior. 2. ed. New York: Macmillan.

Sincerre, B. P., Sampaio, J. O., Famá, R., \& Santos, J. O. (2016). Emissão de Dívida e Gerenciamento de Resultados. Revista Contabilidade \& Finanças-USP, v. 27, n. 72. DOI: 10.1590/1808-057x201601660. 
Stadler, C., \& Nobes, C. W. (2014). The influence of country, industry, and topic factors on IFRS policy choice. Abacus, v. 50, n. 4, p. 386-421. DOI: https://doi.org/10.1111/abac.12035.

Taylor, S. E., \& Brown, J. D. (1988). Illusion and well-being: a social psychological perspective on mental health. Psychological bulletin, v. 103, n. 2, p. 193. DOI: 10.1037/0033-2909.103.2.193.

Wooldridge, J. M. (2012). Introductory econometrics: A modern approach. Nelson Education.

Yoshinaga, C. E., \& Ramalho, T. B. (2014). Finanças comportamentais no Brasil: uma aplicação da teoria da perspectiva em potenciais investidores. RBGN-Revista Brasileira de Gestão de Negócios, v. 16, n. 53, p. 594-615. DOI: http://dx.doi.org/10.7819/rbgn.v16i52.1865.

Young, S. (1999). Systematic measurement error in the estimation of discretionary accruals: an evaluation of alternative modelling procedures. Journal of Business Finance \& Accounting, v. 26, n. 7-8, p. 833-862. DOI: https://doi.org/10.1111/1468-5957.00277. 